\title{
Predator-induced maternal effects determine adaptive antipredator behaviors via egg composition
}

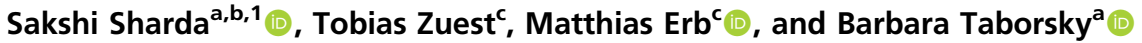 \\ ${ }^{a}$ Department of Behavioural Ecology, Institute of Ecology and Evolution, University of Bern, 3032 Hinterkappelen, Switzerland; ${ }^{b}$ Department of Ecology \\ and Evolution, University of Lausanne, CH-1015 Lausanne, Switzerland; and 'Institute of Plant Sciences, University of Bern, 3013 Bern, Switzerland
}

Edited by Mary Jane West-Eberhard, Smithsonian Tropical Research Institute, Ciudad Universitaria, Costa Rica, and approved June 28, 2021 (received for review August 17, 2020)

In high-risk environments with frequent predator encounters, efficient antipredator behavior is key to survival. Parental effects are a powerful mechanism to prepare offspring for coping with such environments, yet clear evidence for adaptive parental effects on offspring antipredator behaviors is missing. Rapid escape reflexes, or "C-start reflexes," are a key adaptation in fish and amphibians to escape predator strikes. We hypothesized that mothers living in high-risk environments might induce faster C-start reflexes in offspring by modifying egg composition. Here, we show that offspring of the cichlid fish Neolamprologus pulcher developed faster C-start reflexes and were more risk averse if their parents had been exposed to cues of their most dangerous natural predator during egg production. This effect was mediated by differences in egg composition. Eggs of predator-exposed mothers were heavier with higher net protein content, and the resulting offspring were heavier and had lower igf-1 gene expression than control offspring shortly after hatching. Thus, changes in egg composition can relay multiple putative pathways by which mothers can influence adaptive antipredator behaviors such as faster escape reflexes.

maternal effects | developmental plasticity | antipredator response | C-start response | egg size

$\mathbf{N}$ ongenetically transmitted maternal effects can exert modifications in offspring phenotype, leading to individual variation (1) that natural selection can act on (2-4). Maternal effects may act to transfer signals or cues that affect the development of offspring phenotypes persistently (via the deposition of hormones, nutrients, or maternal RNA into eggs), and these effects can even carry over to multiple generations $(5,6)$. It has been hypothesized that via maternal effects in the prenatal stage, mothers can shape offspring phenotype to prepare them for the environment they are likely to encounter after birth $(2,7,8)$. These adaptive maternal effects should only evolve if the mother can reliably forecast the offspring environment $(9,10)$.

Predation risk is one of the most significant environmental factors affecting life history and individual fitness $(11,12)$ as well as population dynamics (13) and social structure $(14,15)$, so one might expect that in dangerous environments mothers prepare their offspring via maternal effects to effectively cope with predation risk. Efficient antipredator behaviors are key for survival because they allow quick and highly flexible responses to unpredictable predator attacks. Therefore, one should expect predatorinduced maternal effects on offspring antipredator behavior to be widespread. However, compared to well-established morphological [e.g., neck helmets in Daphnia (16)] and physiological [e.g., glucocorticoids (17)] maternal effects influencing offspring survival, the adaptive value of maternal effects on offspring behavior is yet unclear (SI Appendix, Table S1) for three main reasons: 1) Previous studies did not evaluate the adaptive value of the reported antipredator behavior in the face of real predation threat (18-21). 2) The changes in offspring behavior induced by maternal exposure to predation threat were either maladaptive $(22,23)$ or they had no detectable effect on predator evasion $(17,24)$. For instance, in three-spine stickleback (Gasterosteus aculeatus), offspring of mothers exposed to predator cues tended to shoal closer together (17) but did so only before but not during or after an attack. Additionally, they were more often victims of predator attacks, probably because they were less vigilant (22), and they had deficits in learning abilities (25). 3) Offspring from predator-exposed mothers may show reduced mobility compared to offspring from unexposed control mothers [e.g., in spider mites, Tetranychus urticae $(23,26)$, and crickets, Gryllus pennsylvanicus (27)]; however, when offspring performed these putatively adaptive antipredator behavior, this involved high opportunity costs, such as reduced foraging time $(15,20)$ (details reviewed in SI Appendix, Table S1).

Thus, despite the general interest in studying maternal effects on antipredator behavior, we are currently lacking clear evidence for adaptive antipredator behavior in offspring that has been induced by maternal effects. Ideally, a test for such effects should focus on behaviors that 1) have been proven to aid predator evasion and 2) do not inflict considerable opportunity costs (e.g., costs on foraging). Fast-start escape responses such as the "C-start" reflexes of fish (28) fulfill these conditions perfectly. These reflexes are very short, in the range of a few seconds to immediately escape the predator, thus inflicting virtually no opportunity costs on timeconsuming vital activities such as foraging. In fish, C-start reflexes are governed by a single action potential determining not only whether an escape response will occur but also the direction of the escape. These reflexes are elicited by the giant Mauthner neurons or "Mcells" located in the hindbrain (29). When the functioning of M-cells in zebrafish larvae prey was experimentally disabled, predators had a three times higher capture success (30). Moreover, in white-tailed damselfish (Pomacentrus chrysurus), out of 18 morphological,

\section{Significance}

In dangerous environments, mothers can prepare their offspring to cope with predation risk. Rapid escape responses such as the characteristic "C-start reflex" of fishes improve survival chances of offspring during predator attacks. We found that mothers exposed to predators during egg maturation, by changes to egg composition, produced offspring with faster C-start reflexes, which is an unequivocal demonstration of an adaptive maternal effect on offspring antipredator behavior. Moreover, compared to the control treatment, eggs of predator-exposed mothers were larger and contained more total protein and resulted in offspring with larger initial body size but reduced expression of a growth gene.

Author contributions: S.S. and B.T. designed research; S.S. performed research; M.E. contributed new reagents/analytic tools; S.S. and T.Z. analyzed data; and S.S. and B.T. wrote the paper.

The authors declare no competing interest

This article is a PNAS Direct Submission.

Published under the PNAS license.

${ }^{1}$ To whom correspondence may be addressed. Email: s.sharda.12@ucl.ac.uk.

This article contains supporting information online at https://www.pnas.org/lookup/suppl/ doi:10.1073/pnas.2017063118/-/DCSupplemental.

Published September 10, 2021. 
physiological, and behavioral parameters, C-start response latencies to an experimental startle stimulus explained best the long-term survival of juveniles (31). However, despite the obvious adaptive value of escape reflexes for prey survival, it is yet unknown whether they can be modulated by maternal effects.

We hypothesized that mothers living in high-risk environments might induce faster C-start reflexes in offspring, which would render unequivocal evidence for adaptive predator-induced maternal effects on offspring behavior. While generally, parental effects may be conveyed to offspring by parental behavior (32-35) or by the modification of gametes $(17,36-38)$, in this study, we hypothesized that the maternal effects on offspring evasion behavior are transmitted through the eggs if mothers are exposed to predator cues during egg maturation. Consequently, we further hypothesized that treatment differences in these offspring will manifest already in the egg and fry stage. We performed two experiments to investigate predator-induced maternal effects on escape behavior and its putative underlying mechanisms in a cooperatively breeding vertebrate, the cichlid fish Neolamprologus pulcher. In these fish, young of recent clutches delay dispersal from the natal territory greatly beyond the time of independence from parental care and help raise the offspring of a dominant breeder pair, thereby often forgoing their own reproduction. Maternal effects enhancing offspring survival and antipredator behavior will primarily increase the direct fitness of parents, but in this species, selection of maternal effects might be further strengthened via positive feedback on maternal fitness because all surviving offspring stay at the natal territory as alloparental brood care helpers at least until they are sexually mature.

If the maternal exposure to predators results in specific antipredator adaptations, we predicted 1) that offspring from predator-exposed female $N$. pulcher will exhibit faster C-start reflex responses (Table 1). When mothers experienced predator cues during egg maturation, 2) mothers should have a higher overall provisioning of their eggs; they should lay larger eggs (17) with a higher total protein content and with more steroids and other lipids owing to their role in offspring growth (39) and antipredator responses (40). 3) Larger eggs should result in larger offspring (41). A larger size of offspring is considered an important adaptation to evade gape-size limited predation (42), which is strong particularly in small juvenile $N$. pulcher, and it enhances the burst swim speed (42). 4) The larger offspring size from parents exposed to predation threat should be accompanied by a higher expression of genes of the somatotropic axis in these offspring (Table 1).

Alternatively, although not mutually exclusively, maternal predator exposure may lead to an enhanced general stress responsiveness in offspring. If this were the case, 5) we should expect offspring to be generally more anxious (e.g., to seek shelter more readily and often; see Table 1). 6) We should also predict a stronger signaling of the stress axis in offspring from parents exposed to predator threat, which would be evident by differential expression of corticosteroid receptor genes in offspring from the two treatment groups (see Table 1).

In a first experiment, we teased apart egg-mediated maternal effects by letting $N$. pulcher pairs produce unfertilized eggs either in the presence or absence of predator exposure. Eggs from the two treatments were compared for weight and total amino acids. In addition, we performed metabolomic fingerprinting, i.e., an untargeted screen of small organic molecules by liquid chromatography-mass spectrometry (LC-MS) to further differentiate maternal investment in these eggs. In a second experiment, we compared offspring from predator-exposed and nonexposed parents. We collected broods from the two treatments and raised them without parents. In the fry stage (first 2 to $3 \mathrm{wk}$ of life), we analyzed treatment differences in growth, expression of key genes in the somatotropic and stress axes, and offspring survival. Subsequently, at an age of 3 mo (juvenile stage), we performed a predator escape task to measure the latency to show C-start escape reflexes and the behavior after recovery when fish reemerged after the escape response. Finally, at the age of $4 \mathrm{mo}$, we measured the behavioral responses toward video presentations of predators.

\section{Results}

\section{Experiment 1.}

Size and composition of unfertilized eggs. Females in the predator treatment laid eggs with a higher mean wet weight (Table 2 and Fig. 1A), albeit with no difference in clutch sizes between treatment. Furthermore, they laid eggs with a higher net protein content (Table 2 and Fig. $1 B$ ), but eggs did not differ in protein concentration between treatments (Table 2), suggesting that protein content was not adjusted to treatment. Accordingly, total protein content of eggs was correlated with egg weight (Pearson's correlation coefficient $r=0.74, P<0.001)$. Metabolomic fingerprinting identified a total of 519 unique mass features. None of them differed between treatments after correcting for multiple testing (SI Appendix, Table S7 shows 24 features with $>2$-fold differences between treatments and a difference of $P<0.05$ before multiple testing correction).

\section{Experiment 2.}

Clutch size and fry stage. Similar to experiment 1 , clutch sizes from the two parental treatments (predator or control) did not differ in experiment 2 (Table 2 and SI Appendix, Fig. S2). However, the

Table 1. Predictions for the different behavioral and physiological assays performed in both experiments of our study

Number in text Category Trait Predictions for offspring from predator-exposed parents

Antipredator adaptations

(1) Behavior

(2) Egg trait

(3) Size/growth

(4)

Gene

expression

General stress responsiveness

(5) Behavior

(6)

Gene

expression

Onset of C-start reflex Egg weight

Total protein content

Metabolite composition

Offspring size at fry stage Growth until 3 mo

Expression of three key somatotropic genes: igf-1, ghr, and igf-2

Seek shelter

Expression of $g r$
Faster

Higher (17)

Higher (39)

Higher deposition of steroidal compounds and metabolites related to the somatotropic and stress axis (17)

Larger $(41,100)$

Faster (100)

Higher (indicating higher growth) (100)
More readily immediately after threat and generally more often
Lower, as it is involved in negative feedback on
hypothalamic-pituitary-adrenal/interrenal (HPA/HPI) stress responses (101) 
Table 2. Model results presented with estimates and SE for each factor

\begin{tabular}{|c|c|c|c|c|c|}
\hline Final model & Estimate $\pm \mathrm{SE}$ & $t / z$ & $P$ & $\mathrm{~N}_{\text {predator }}$ & $\mathrm{N}_{\text {control }}$ \\
\hline \multicolumn{6}{|l|}{ A. Experiment 1} \\
\hline \multicolumn{4}{|l|}{ Fresh egg weight ( 5 replicates per brood) } & \multirow[t]{3}{*}{5} & \multirow[t]{3}{*}{6} \\
\hline Intercept & $8.63 \mathrm{e}^{-04} \pm 2.38 \mathrm{e}^{-05}$ & 36.17 & & & \\
\hline Treatment & $-1.55 \mathrm{e}^{-04} \pm 3.54 \mathrm{e}^{-05}$ & 4.39 & 0.001 & & \\
\hline \multicolumn{4}{|l|}{ Clutch size } & \multirow[t]{3}{*}{5} & \multirow[t]{3}{*}{6} \\
\hline Intercept & $79.83 \pm 10.17$ & 7.85 & & & \\
\hline Treatment & $-12.83 \pm 15.08$ & -0.85 & 0.35 & & \\
\hline \multicolumn{4}{|c|}{ Total amino acid content ( 3 replicates per brood) } & \multirow[t]{3}{*}{5} & \multirow[t]{3}{*}{6} \\
\hline Intercept & $212.26 \pm 5.56$ & 38.13 & & & \\
\hline Treatment & $-19.97 \pm 5.56$ & -3.59 & 0.001 & & \\
\hline \multicolumn{3}{|c|}{ Amino acid concentration ( 3 replicates per brood) } & & \multirow[t]{3}{*}{5} & \multirow[t]{3}{*}{6} \\
\hline Intercept & $0.42 \pm 0.008$ & 48.71 & & & \\
\hline Treatment & $-0.007 \pm 0.008$ & -0.89 & 0.33 & & \\
\hline \multicolumn{6}{|l|}{ B. Experiment 2} \\
\hline \multicolumn{6}{|l|}{ Clutch size and fry stage } \\
\hline \multicolumn{4}{|l|}{ Clutch size } & \multirow[t]{6}{*}{8} & \multirow[t]{6}{*}{10} \\
\hline Intercept & $59.17 \pm 11.94$ & 16.65 & & & \\
\hline Treatment & $16.94 \pm 12.12$ & 1.39 & 0.53 & & \\
\hline Mean centered maternal weight & $4.03 \pm 2.62$ & 1.53 & 0.08 & & \\
\hline Number of presentations & $0.04 \pm 0.64$ & 0.06 & 0.31 & & \\
\hline Treatment $\times$ number of presentations & $-1.03 \pm 0.64$ & -1.61 & 0.07 & & \\
\hline \multicolumn{3}{|c|}{ Dry body weight at day 3 ( 2 to 4 replicates per brood) } & & \multirow[t]{4}{*}{8} & \multirow[t]{4}{*}{10} \\
\hline Intercept & $3.57 e^{-04} \pm 4.25 e^{-04}$ & 8.40 & & & \\
\hline Treatment & $-3.46 \mathrm{e}^{-04} \pm 1.66 \mathrm{e}^{-04}$ & -2.08 & 0.05 & & \\
\hline Density of larvae at day 3 & $-8.98 \mathrm{e}^{-07} \pm 9.05 \mathrm{e}^{-07}$ & -0.99 & 0.33 & & \\
\hline \multicolumn{3}{|c|}{ Dry body weight at day 10 ( 3 to 4 replicates per brood) } & & \multirow[t]{4}{*}{8} & \multirow[t]{4}{*}{10} \\
\hline Intercept & $6.42 \mathrm{e}^{-04} \pm 8.75 \mathrm{e}^{-05}$ & 7.33 & & & \\
\hline Treatment & $1.50 \mathrm{e}^{-05} \pm 4.13 \mathrm{e}^{-05}$ & 0.36 & 0.71 & & \\
\hline Density of larvae at day 10 & $-5.60 \mathrm{e}^{-06} \pm 2.22 \mathrm{e}^{-06}$ & -2.52 & 0.02 & & \\
\hline \multicolumn{6}{|l|}{ Predator escape test } \\
\hline \multicolumn{2}{|l|}{ Response time (3 replicates per brood) } & & & 8 & 10 \\
\hline Intercept & $-0.008 \pm 0.10$ & -0.82 & & & \\
\hline Treatment & $0.038 \pm 0.019$ & 2.01 & 0.04 & & \\
\hline Angle with feeding plate & $0.00055 \pm 0.00053$ & 1.03 & 0.28 & & \\
\hline Length & $-0.044 \pm 0.049$ & -0.90 & 0.35 & & \\
\hline Feeding frequency (3 replicates per brood & & & & 8 & 10 \\
\hline Intercept & $0.18 \pm 0.44$ & $0.41^{z}$ & & & \\
\hline Treatment & $0.58 \pm 0.25$ & $2.29^{2}$ & 0.019 & & \\
\hline $\mathrm{FI}$ & $-11.31 \pm 12.75$ & $-0.88^{z}$ & 0.16 & & \\
\hline
\end{tabular}

When a model yielded $z$ values, this is indicated with a superscripted " $z$." Information on model fixed and random factors are given in SI Appendix, Table S6. $P$ values $<0.05$ are formatted in bold; values of $0.05<P<0.1$ are formatted in italics (except intercepts). $\mathrm{N}_{\text {predator }}$ and $\mathrm{N}_{\text {control }}$ represent the number of broods, each produced by a different breeding pair. Estimates are based on sum contrasts, where the intercept represents the overall mean for the two factors (control and predator) in this study. Estimates for treatment refer to that of the control treatment. All other model factors are continuous covariates.

number of video presentations parents received before spawning tended to interact with clutch size (Table 2). Visual inspection of the interaction suggests that clutch size tended to increase with the number of presentations in the predator treatment, which is in contrast to the control treatment (SI Appendix, Fig. S3).

In fry at experimental day 3 , we measured three key genes from the somatotropic axis (insulin-like growth factors, igf-1 and $i g f-2$, and growth hormone $[\mathrm{GH}]$ receptor, $g h r)$ and two from the stress axis (glucocorticoid receptor, $g r$, and mineralocorticoid receptor, $m r)$. Among these, the relative expression of igf- 1 was on average $35 \%$ lower in offspring of parents receiving the predator treatment as compared to the control treatment (Fig. $1 C$ and Table 3), whereas there was no difference in the relative expression of igf- 1 at day 10 (Fig. $1 D$ and Table 3 ). The expression of the $g h r$ and igf-2 genes did not differ between treatments on either experimental day (Table 3 and SI Appendix, Table S5, respectively) and neither did the $g r$ and $m r$ genes from the stress axis (SI Appendix, Table S5). The expression of some genes of the stress and somatotropic axes was positively or negatively correlated (SI Appendix, Fig. S4), albeit with no treatment differences.

Offspring dry weight (Table 2, B) was higher in predatortreated offspring compared to control offspring at day 3 but not at day 10. The proportion of surviving offspring hatched from the two parental treatments (generalized linear mixed model [GLMM], $n=23$; day 3: treatment: $\mathrm{z}=-0.29, P=0.76$, clutch size: $\mathrm{z}=-0.15, P=0.87$; day 10 : treatment: $\mathrm{z}=-0.40, P=0.66$, clutch size: $\mathrm{z}=-0.025, P=0.98$, respectively; SI Appendix, Table S4) did not differ at days 3 and 10.

Predator escape test. At the age of $3 \mathrm{mo}$, the experimental offspring were exposed to a standardized threat stimulus, a dropping marble mimicking a darting attack of a fish predator, while they were foraging at a food patch (cf. Materials and Methods). The latency to elicit a C-start reflex in response to the dropping 

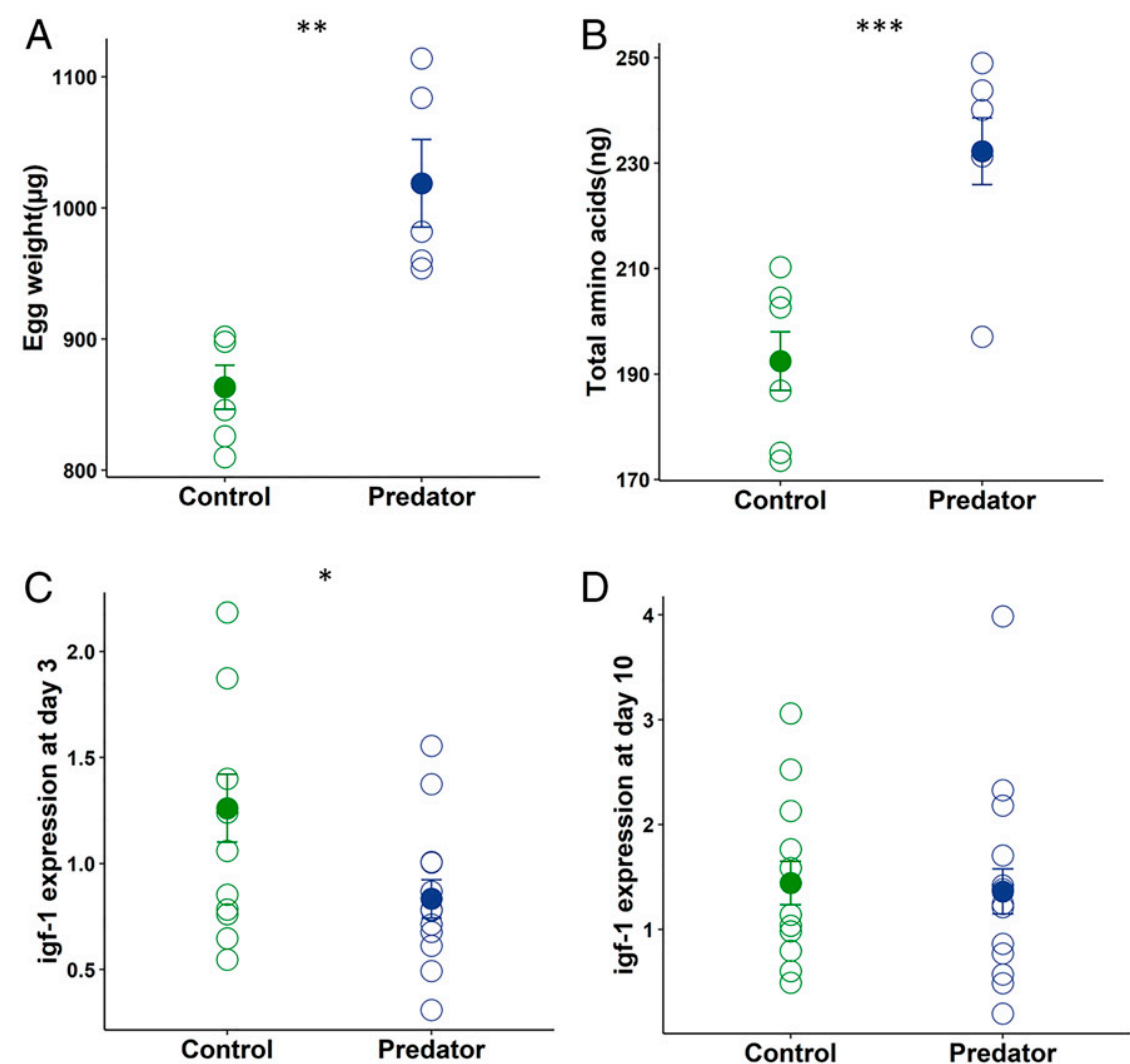

Fig. 1. Clutch characteristics of unfertilized eggs from the two parental treatments in experiment 1, and clutch size and gene expression of igf-1 in experiment 2. (A) Fresh egg weights measured right before amino acid quantification. Open circles: mean of five unfertilized eggs obtained per brood; solid circles and error bars: means \pm SE across broods; $\mathrm{N}_{\text {control }}=6$ (two data points overlap) and $\mathrm{N}_{\text {predator }}=5$ for each treatment. (B) Total amino acids present in eggs. Open circles: mean of three eggs per brood; solid circles and error bars: means \pm SE across broods. (C) Relative expression of igf-1 gene expression at day 3 (with respect to the reference gene $r p / 13 a$ ) and $(D)$ day 10 of experiment 2 . In the statistical models, igf- 1 values on day 3 were subjected to a negative power transformation using the boxcox function, whereas those on day 10 were subjected to a positive power transformation. Here, the means (solid circles) and SE and individual data points (open circles) are back-transformed to their original scale. ( $A-D)$ Blue: control treatment; green: predator treatment; bars show mean $\pm \mathrm{SE} ; * * * P<0.001, * * P<0.01, * P<0.05$.

marble was significantly shorter in offspring from predatorexposed than control parents (Table 2 and Fig. $2 A$ ).

There was no treatment effect on the likelihood of offspring to seek refuge in the shelter immediately after the marble drop or on the frequency of shelter visits in the 15 min following the marble drop (SI Appendix, Table S4). However, offspring from predator-treated parents showed a reduced frequency of visiting the food patch after recovery (Table 2 and Fig. $2 B$ ). Moreover, at the age of $4 \mathrm{mo}$, we tested if offspring from the two treatments responded differently toward visual and chemical predator cues (SI Appendix) to test for maternally induced fear responses against predators, but the two treatments did not differ in the likelihood to leave the safe zone of the tank and in alertness behavior (SI Appendix, Table S4).

\section{Discussion}

Here, we showed that parental exposure to a natural predator induced changes in egg composition, accelerating the release of C-start reflexes in 3-mo-old $N$. pulcher offspring, which is a key adaptation in fish to survive the strike of a predator $(30,43)$, enhancing their long-term survival (31). In our study, the change in the reflex behavior can only be caused by differences in egg composition between treatments because the eggs of experimental parents were removed immediately after spawning and all offspring were reared under the same conditions, separately from parents and without any predator cues. Offspring from the predator treatment released the C-start reflex on average $50 \mathrm{~ms}$ faster than offspring of the control treatment, suggesting that egg-mediated maternal effects altered the functioning of the giant Mauthner neurons controlling this reflex (30). Moreover, offspring from the predator treatment had a lower feeding frequency after recovering from the simulated threat, possibly indicating higher risk aversion in these individuals.

We studied putative mechanisms underlying this maternal effect by analyzing unfertilized eggs (experiment 1 ) and assaying gene expression of fry (experiment 2). Our analysis revealed that in response to predator exposure, mothers differentially allocated nutrients leading to changed egg composition: predatorexposed mothers produced larger eggs, which contained a higher total protein content. The metabolomic profiles did not differ significantly between eggs of the two treatments. A larger egg size generally increases offspring size in fish (41, 44-46). As expected, at almost $2 \mathrm{wk}$ after spawning, that is, $3 \mathrm{~d}$ after reaching the freeswimming stage, offspring from predator-exposed parents were larger compared to their control counterparts, which was accompanied by a lower expression of igf-1, a gene coding for a key hormone of the somatotropic axis.

Antipredator Behaviors. Predator cues are known to enhance escape responses of fish $(47,48)$. Our finding that juvenile offspring from predator-exposed $N$. pulcher mothers exhibit faster $\mathrm{C}$-start responses is noteworthy for three reasons. First, it reveals the existence of maternally induced developmental plasticity on a reflex behavior, something that has not previously been explored. 
Table 3. Gene expression estimates and SE of the three key somatotropic genes tested at experimental day 3 and day 10

\begin{tabular}{lccccc} 
& Estimate & $\mathrm{t}$ & $P$ & $\mathrm{~N}_{\text {predator }}$ & $\mathrm{N}_{\text {control }}$ \\
\hline $\begin{array}{l}\text { Day } 3 \\
\text { igf-1 }\end{array}$ & & & & & \\
$\quad$ Intercept & $1.0094 \pm 0.0053$ & 188.2 & & 11 & 10 \\
$\quad$ Treatment & $-0.011 \pm 0.0053$ & -2.1 & 0.035 & & \\
$\begin{array}{l}\text { igf-2 } \\
\quad \text { Intercept }\end{array}$ & $1.15 \pm 0.06$ & 17.65 & & 11 & 10 \\
$\quad$ Treatment & $0.07 \pm 0.06$ & 1.18 & 0.22 & & \\
ghr & & & & 11 & 10 \\
$\quad$ Intercept & $0.99 \pm 0.0068$ & 146.85 & & & \\
$\quad$ Treatment & $0.00019 \pm 0.0068$ & -0.03 & 0.99 & & \\
$\begin{array}{l}\text { Day 10 } \\
\text { igf-1 }\end{array}$ & & & & 12 & 11 \\
$\quad$ Intercept & $1.02 \pm 0.03$ & 27.46 & & & \\
$\quad$ Treatment & $0.0059 \pm 0.037$ & 0.15 & 0.86 & & \\
igf-2 & & & & 12 & 11 \\
$\quad$ Intercept & $1.018 \pm 0.017$ & 59.13 & & & \\
$\quad$ Treatment & $-0.021 \pm 0.016$ & -1.29 & 0.19 & & \\
ghr & & & & 12 & 11 \\
$\quad$ Intercept & $1.0068 \pm 0.012$ & 79.84 & & & \\
$\quad$ Treatment & $-0.0094 \pm 0.012$ & -0.75 & 0.42 & & \\
\hline
\end{tabular}

$P$ values $<0.05$ are highlighted in bold. Estimates are box cox transformed, and all models accounted for brood ID as a random factor (see SI Appendix Table S6 for details on transformations). Estimates are based on sum contrasts, where the intercept represents the overall mean of each factor. Estimates for treatment refer to that of the control treatment and represent transformed values used in the models.

Second, it suggests an important role of nongenetic maternal effects on the neural organization of the offspring brain, i.e., on the M-cells controlling this reflex (30). Third, and most importantly, it shows the existence of adaptive predator-induced maternal effects on offspring behavior. Faster C-start responses are a specific adaptation to lifethreatening darting attacks from the water column, which is the main hunting strategy of the major predator Lepidiolamprologus elongatus.

C-start reflexes involved in response to sudden threats are controlled by a pair of large reticulospinal neurons, the M-cells, and their associated excitatory and inhibitory networks. The M-cells elicit a fast (around $10 \mathrm{~ms}$ ) muscle reflex contraction, enabling the body to bend away from the direction of a predator attack in a "C-shape" fashion $(29,49)$. We hypothesized that the reduced response latency of offspring from predator-exposed mothers was caused by an increased excitability of the M-cells. $\mathrm{M}$-cells are solely responsible for initiating the fast-start reflex responses, as their ablation leads to the loss of these reflexes (30), pinning down the importance of these neurons. The responses initiated by M-cells can be modulated, for instance, in response to social cues (28). Outside a predatory context, maternal effects have been shown to modify neuronal excitability, for instance, in the hippocampus of rats (32), which led to changes in the neuroanatomy of the offspring brain, an effect detectable even two generations downstream. Parental effects can be transferred through male or female gametes $(36,50)$. In our study, parental predator exposure led to intergenerational transfer of ecologically relevant information through the gametes, resulting in a behavioral adaptation of offspring.

In addition to responding faster to the startle stimulus, offspring of predator-exposed mothers also visited the food patch less often after the startle response, possibly indicating that they were more risk averse. In three-spined stickleback (G. aculeatus), offspring from predator-exposed mothers show reduced orientation toward a predator, a behavior proposed by the authors to be rather maladaptive (22), indicating that maternal effects on longterm behavior toward predators are not always adaptive. In contrast, in our study species $N$. pulcher, the reduced frequency to visit the feeding patch after a simulated attack occurred near this patch should provide a survival advantage in the presence of predators, although under natural conditions this might result in a loss of feeding opportunities $(41,51,52)$ (SI Appendix, Table S1).

Putative Mechanisms of Maternal Effects on Antipredator Behaviors. Egg composition. In our study, predator-exposed females of $N$. pulcher laid heavier eggs compared to no-predator control females, whereas clutch size did not differ, which is in line with previous findings in three-spined sticklebacks $(17,22)$ and the mouthbrooding cichlid, Eretmodus cyanostictus (46). Moreover, a previous study found that $N$. pulcher produce larger eggs, again without accompanying changes in clutch size, when social group size is experimentally reduced (53). This was interpreted as an antipredatory parental effect because predation risk strongly increases with decreasing group size in this species (54). In fish, larger eggs typically result in larger larvae (41, 44-46), which is what we also observed in our study. Taken together, our results suggest that under higher perceived predation risk, females increase their egg size to produce larger offspring, thereby providing them with a size and mobility advantage in the vulnerable first days after hatching $(41,42)$. In fish, larger body size is important for predator evasion, especially at the earliest life stages
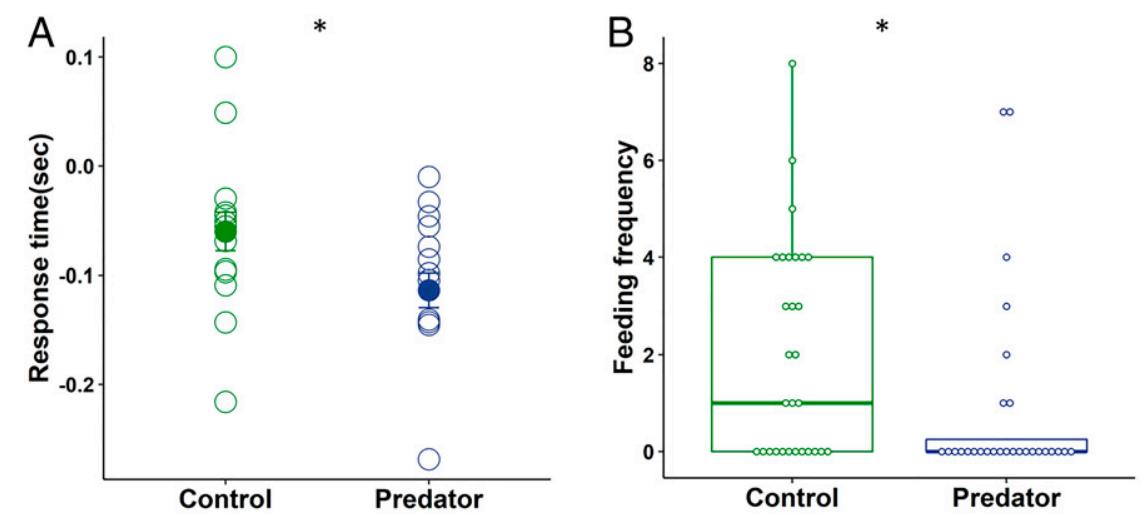

Fig. 2. Behaviors assayed in the predator escape test in experiment 2. (A) Time until fish responded to the dropping marble by a C-start reflex. Time point "0" represents the instant the marble touches the water; most fish showed a negative latency, meaning that they responded to the marble before time point 0 ; open circles: single data points; solid circles and error bars: mean \pm SE (see Movie S1). (B) Number of times the test fish visited the feeding plate during 15 min after the marble had been dropped. Open circles: single data points; box plots show medians, interquartile ranges; whiskers represent values in between the upper quartile and maximum value. $\left(A\right.$ and $B$ ) Blue: control treatment; green: predator treatment; ${ }^{\star} P<0.05$. 
$(55,56)$. Since most predators are gape-size limited fish species, a larger size of potential prey confers survival benefits. Moreover, a larger body size enhances the mobility of small offspring (42). Because our study species faces very high predation threat in the field $(14,57)$, maternal effects increasing larval size might be a key adaptation.

The production of larger eggs is likely to impose long-term costs, and it is therefore possible that this is a strategic decision of the females. In soil mites (Sancassannia berlesei) (5) and the live-bearing fish Gambusia holbrooki (58), the production of larger eggs correlated negatively with female fecundity. In contrast, in species where an egg size-fecundity trade-off is not observed, like in reef fish (59), cichlids $(46,53)$, and sticklebacks $(17,22)$, the production of larger eggs may be traded off against future survival and reproduction (53).

The larger eggs of predator-exposed females in our study also contained a higher absolute protein amount, suggesting that the production of larger eggs is more costly for females, at least at the level of protein provisioning. Females invested more in eggs by a quantitative enrichment of proteins rather than by strategically adjusting protein content, indicating that like in other fish $(41,60)$, larger N. pulcher eggs are of higher quality. Egg proteins deposited in yolk and eggshell are the major constituents of an egg (61). Yolk proteins are derived from maternal liver cells, and the production of liver proteins is costly, making up around $80 \%$ of oxygen consumption in isolated hepatic cells of fish (62). Parental experience may also be passed on to offspring via subtle alteration of metabolites in egg provisioning. For instance, in red drum (Sciaenops ocellatus), predator-induced maternal investment of certain essential fatty acids such as arachidonic acid and docosahexaenoic acid led to faster larval escape behavior (40). However, in our study, we could not detect differences between the metabolomics profiles of the two treatments.

Growth and somatotropic gene expression. Initial egg size differences (see Fig. 1) between treatments translated into differential offspring dry weight at day 3; offspring from predator parents were heavier than their control counterparts. This maternal effect was short-lived; it had vanished by day 10 of the experiment, and accordingly, there were also no long-term size differences at an age of 3 mo. Thus, offspring size differed shortly after birth as a consequence of the larger egg size (41), but growth did not differ between treatments later on in the juvenile stage. While this means that juveniles of predator-exposed mothers had no size advantage left at 3 mo of age, it also means that the behavioral differences between treatments, the C-start response and the feeding frequencies, cannot be simply explained by the current size when behaviors were tested.

As predicted, maternal predator exposure affected gene expression of the somatotropic axis. However, opposite to our prediction, igf-1, a key growth factor in muscle and skeletal growth and in metabolism (63), showed higher expression in the control group at day 3. Like offspring size differences, this maternal effect was short-lived and had vanished by day 10 . The higher expression of igf- 1 in the control group relative to the predator treatment present at day 3 suggests that either 1) control fish were able to grow faster after day 3 or 2) fish from the predator treatment, which were larger at day 3 , grew more slowly after day 3 , such that both treatment groups reached similar sizes at day 10. Regarding the first scenario, "catch-up growth" to compensate for hatching from a smaller egg resulting quickly in equal body sizes has previously been reported in salmonids $(64,65)$ and in mouthbrooding cichlids (66). However, the alternative scenario occurred in mouthbrooding cichlids (18), in which offspring of predatorexposed mothers first grew faster, followed by a period of slower growth ("decompensatory growth"), although in the mouthbrooders, growth differences were not related to egg size.

The fact that we find short-lived maternal effects on body size and differential igf- 1 expression shortly after birth, but not afterward, also suggests that neuronal programming altering the speed of the C-start response occurs early in life, which is the time window when the fish brain develops (67). Moreover, beyond growth, IGF-1 has multiple functions, including risk-averse behavior and foraging (68-70). Therefore, it is possible that the regulation of the somatotropic axis early in life may have influenced later escape responses. Thus, we propose that future work should focus on the neurodevelopment of the earliest life stages when investigating the neural mechanisms underlying the plasticity of C-start reflexes.

General stress response. We can safely reject the alternative hypothesis that the maternal effects reported here are due to a generally increased stress responsiveness elicited in offspring of predator-exposed mothers. If offspring of exposed mothers were more stressed or anxious, we should have found maternal effects also on shelter use and hiding behavior of offspring after they experienced a threat, which was not the case. Maternal effects influence the C-start reflex in response to a simulated attack and behavior at the place of the attack (i.e., at the feeding dish) but not anywhere else in the tank (Table 1). They did also not influence the likelihood to leave the safe zone or to show alertness behavior when presented the view of $L$. elongatus predators (SI Appendix, Table S4). N. pulcher juveniles, even the smallest ones, respond very specifically to different types of predators, for instance, by contributing to active defense against predators of eggs, larvae, and young (71-74). This indicates that behavioral responses are tailored against predators rather than reflecting generalized anxiety.

Furthermore, a change in stress responsiveness of offspring induced by the maternal treatments would also have been indicated by effects on the expression of stress axis genes (75). However, there were no differences in the expression of the two stress axis genes $\mathrm{grl}$ and $m r$ between treatments.

\section{Conclusions}

In our study, we provide unequivocal evidence of an adaptive predator-induced maternal effect on offspring behavior because C-start reflexes are vital to escape the strike of predators (30) and bear no significant opportunity costs. We further show that this nongenetic maternal effect was mediated by changes in egg composition because eggs were hatched and reared in the same environmental conditions and in the absence of parents. Indeed, we detected a suite of differences between the predator and control treatments in unfertilized eggs, which indicate higher investment in egg quality by predator-exposed mothers. A higher quality of eggs might be causally involved in the effect on escape responses. Potential additional mechanisms, such as the deposition of particular hormones $(17,27,38)$ or maternal RNAs $(76)$ in eggs, warrant future study. While previous work on predatorinduced maternal effects showed changes in anxiety and riskaversive behaviors of offspring, here, we show that maternal effects via egg composition increase the escape response competence toward predator attacks.

\section{Materials and Methods}

Study Species and Housing Conditions. The cooperatively breeding cichlid $N$ pulcher and its natural predator, L. elongatus, are both endemic to Lake Tanganyika, East Africa (77). N. pulcher social groups consist of a breeder pair with related and unrelated subordinate individuals called helpers, who perform alloparental brood care (78). It is a well-suited model to study predator-induced parental effects because 1) predation pressure in the wild is very high $(14,57)$, and this significantly affects the survival probability of group members, especially of small subordinate helpers and offspring (54). This high predation pressure has driven the evolution of sociality in this species (14). 2) Parental effects in eggs have been reported in this species (53), but it is still not known if these are implicated in predation evasion behavior of offspring.

Predator-Video Presentations in Experiments 1 and 2. N. pulcher can distinguish between conspecifics and predators when presented with their animated pictures (48). Therefore, to emulate predation risk, videos (SI Appendix, 
Materials and Methods for video compilation methods) of $L$. elongatus were shown to $N$. pulcher breeder pairs of both experiments twice daily, $3 \mathrm{~d}$ a week during the egg maturation period, while for the control treatment, a blank aquarium was presented (number of presentations in experiment 1, control treatment: mean $=15.6$, range $=12$ to 19 ; predator treatment: mean $=19.25$, range $=12$ to 20 ; experiment 2 , control treatment: mean $=19.4$, range $=8$ to 30; predator treatment: mean $=17.25$, range $=10$ to 22 ) at randomized times of the day to prevent habituation. Any clutches females laid before they received 2 wk of video presentations were discarded because $N$. pulcher females need at least 2 wk to produce a new clutch. However, intervals between clutches can be much longer. Previous work showed that a blank aquarium is indeed an appropriate control in this species $(48,79)$. N. pulcher can distinguish different species of animated fish pictures [they show a stronger response to predators and conspecifics than to herbivorous fish $(48,80)]$, and they also can distinguish pictures and videos of fish from displays of a moving object or a nonmoving background $(48,79)$ while showing equally low responses to a moving object and a nonmoving background (as presented in our study). This indicates that a nonmoving background is an appropriate control, as $N$. pulcher can distinguish moving predators from other moving objects.

Along with visual cues, we also added chemical cues from the predators during the presentation by injecting $12 \mathrm{~mL}$ of water from the predatorholding tank. In our study species, olfactory and visual predator cues are equally important (80), which is why we used a combination of these cues.

\section{Experimental Design.}

Experiment 1. To exclude effects of embryonal metabolism on egg composition, we obtained and assayed unfertilized eggs (SI Appendix, Materials and Methods and Fig. S1). Eleven breeding pairs, five from the predation threat treatment and six from the control treatment, were presented with videos and chemical cues as described until spawning a clutch. Maternal $(t=-0.698$, $P=0.44)$ and paternal $(\mathrm{t}=0.01, P=0.99)$ weights did not differ systematically between control and predator treatments. We collected the unfertilized clutch and measured individually five randomly chosen eggs to the nearest microgram to obtain wet weights. Three further eggs were randomly chosen and preserved at $-80^{\circ}$ for 3 mo until processed for total amino acid analysis by acid hydrolysis followed by phenylisothiocyanate coupling and reverse phase high performance liquid chromatography (HLPC) (81) (SI Appendix). Finally, up to 15 randomly chosen eggs were flash frozen in liquid nitrogen and stored at $-80^{\circ}$ for later metabolomics analysis. Of these, 10 eggs of each brood were subjected to metabolomic fingerprinting by high-resolution ultra-high pressure liquid chromatography coupled to mass spectrometry (UHPLC-MS) (S Appendix, Materials and Methods). Metabolomic fingerprinting is an untargeted global analysis of small molecular weight metabolites (50 to 1,200 Da) that can identify treatment differences between metabolic products, which, for example, may be derived from steroid hormones deposited in eggs.

Experiment 2. Also, in this experiment, maternal $(\mathrm{t}=-1.182, P=0.25)$ and paternal ( $\mathrm{t}=-1.598, P=0.13$ ) weights did not differ systematically between control and predator treatments. To avoid embryonal exposure to the predator treatment, eggs laid by breeding pairs in this experiment $(n=13$ predator and $n=14$ control clutches) were removed from experimental tanks as soon as they were detected ( $\leq 24 \mathrm{~h}$ after spawning) and reared separately to the free-swimming stage (SI Appendix). Afterward, all fry of a brood were transferred together to individual 20-L tanks for further rearing; they received a standardized feeding regime (SI Appendix).

At days 3 and 10 (Fig. 3A), all surviving fry were removed from the tank, placed in a white container, and counted. Before placing them back in their home tanks, we randomly sampled four to eight fry (eight fry were sampled from clutches of $>30$ eggs and four from clutches of $<30$ eggs) using random numbers previously generated in $\mathrm{R} 2.14 .1$. The shoal was counted starting alternately from the left or right side of the collection container, and the fry in the position of the random number was caught and euthanized with an overdose of MS222; two to four of them were preserved in Roti-Histofix 4\% for dry weight measurements. The remaining two to four fry were preserved in RNAlater (at $4{ }^{\circ} \mathrm{C}$ for $1 \mathrm{wk}$ and then to $-80{ }^{\circ} \mathrm{C}$ until assaying) for gene expression assays.

Escape test. At day $83( \pm 3 \mathrm{~d})$, three fish from each brood $(n=80$ individuals from 27 clutches; one fish died a day after being transferred to the experimental tank) were randomly selected to evaluate their ability to escape from a sudden, threatening stimulus, a dropping marble $(18,80)$ (for setup, see Fig. 3B; see SI Appendix, Materials and Methods for detailed procedures and Movie S1). The sex of the experimental fish was unknown, as at this age the gonads and genital papillae are not yet differentiated, and the sex of $N$. pulcher cannot be determined visually. The marble drop method has been used previously to emulate sudden predator attacks during experiments in our study species $(80,82)$. It perfectly simulates extremely rapid, unpredictable darting attacks that the major predator $L$. elongatus makes from the water column

Behavioral analyses from video recordings were done with the observer (S.S.) being blind to parental treatment (i.e., predator or control) on VLC Media Player and ImageJ 1.47v (https://rsb.info.nih.gov/ij). Four parameters were measured: 1) response latencies to the dropping marble: difference between the instant the marble touched the water and the onset of retraction of the focal fish from the feeding dish, measured with the software BORIS (83) by slowing the video by $10 \times ; 2$ ) termination of flight response: whether the flight response terminated by entering or by stopping right before the shelter; 3) frequency of entering the shelter during $15 \mathrm{~min}$ after
A

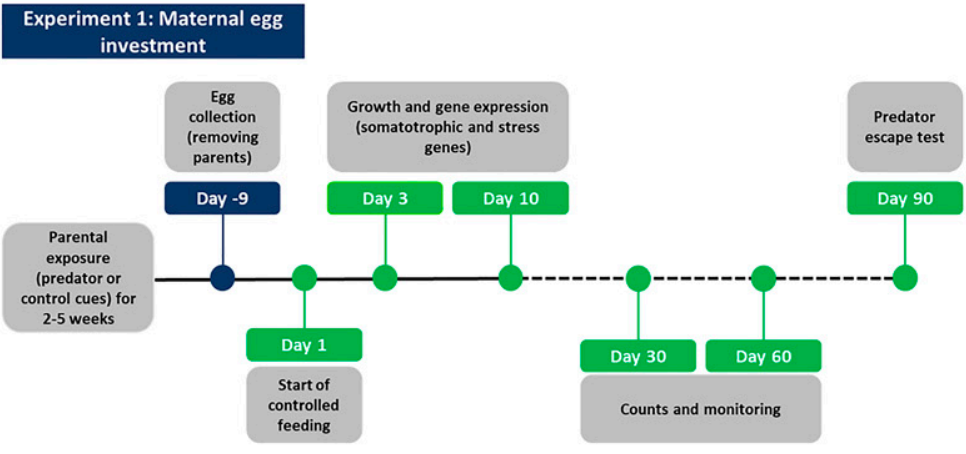

Experiment 2: Offspring growth and anti-predator escape responses

B

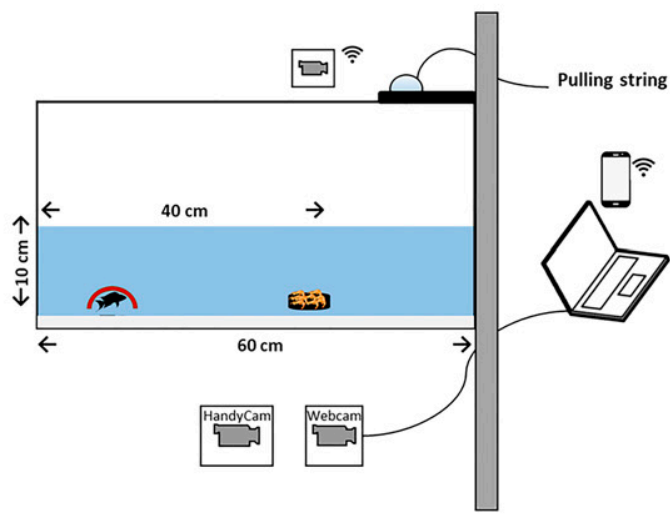

Fig. 3. Experimental timeline and design for the predator escape test. ( $A$ ) Timeline of experiments 1 (in blue) and 2 (in green) from exposure of parents to predator cues to the escape test. (B) Predator escape test (performed at day 90): a 200-L tank was virtually subdivided along its long axis into three vertical zones: a "shelter zone" with the shelter placed $15 \mathrm{~cm}$ away from the shorter edge of the tank, a $25-\mathrm{cm}$ feeding zone with a feeding Petri dish placed at the edge of the boundary away from the shelter, and a $20-\mathrm{cm}$ zone where a marble was dropped in a distance of $10 \mathrm{~cm}$ from the petri dish. White sand was used to provide sufficient contrast between a small focal fish and its background on the video recordings. A wooden "marble holder" was fitted and a needle was inserted to hold it in place. The needle, in turn, was attached to a string that could be pulled by the observer. The gray curtain separated the observer from the experimental setup, and the experiment was carried out as described in Escape test. After an acclimatization period, a few pieces of krill were placed on the feeding dish, and the observer waited for the fish to come to feed. The cameras were switched on, and the observer waited for the fish to take up the start position. As soon as the focal fish was spotted feeding on the dish, the needle, supporting the marble, was immediately pulled (within 1 to $2 \mathrm{~s}$ ), and video recording was continued for $15 \mathrm{~min}$ after the marble drop. 
the marble was dropped; and 4) feeding frequency: number of times the focal fish moved from the sheltering zone $(25 \mathrm{~cm}$ away from the feeding plate) to feed from the feeding plate in $15 \mathrm{~min}$.

Body size measurements. Of the fry collected and preserved on days 3 and 10 (two to four replicates per brood), dry weight was determined 6 to 8 mo after being preserved in Roti-Histofix $4 \%$. Before taking fresh weights, any excess fixative was carefully removed by tapping the individual on paper towels. The juveniles were subsequently dried at $60^{\circ}$ for $12 \mathrm{~h}$ and weighed to the nearest microgram

Gene expression analysis. We measured the expression of five candidate genes in the vertebrate somatotropic and stress axes. The vertebrate growth or somatotropic axis includes the GH and its receptor (GHR) and insulin-like growth factors (IGF) I and II. An up-regulation of $g h$ or $g h r$ genes may result in enhanced foraging activity and growth in fish $(66,84)$. For instance, higher $g h r$ gene expression in offspring hatched from smaller eggs was associated with faster growth rates in mouthbrooding cichlids (66). IGF-1 and IGF-2 play a role in muscle activation (85) and are regulated by GH (86).

The mineralocorticoid receptor (MR) is a corticosteroid receptor of the fish hypothalamic-pituitary-interrenal (HPI) stress axis, the function of which depends on where it is expressed in the cell. Membrane MR determines the threshold or sensibility of the limbic stress response system, whereas nuclear MR boosts the initial stress responses and thus is involved in the ability of fish to respond to stressors such as predators. GR1 (glucocorticoid receptor 1) is activated only by higher levels of cortisol after a stress response has been mounted. It modulates stress responses in different tissues $(87,88)$, and through negative feedback on cortisol excretion, it contributes to the termination of stress responses. In $N$. pulcher, the expression of the gr 1 gene is permanently altered by early-life social experience $(89,90)$.

We determined the expression of the somatotropic genes igf-1, igf-2, and $g h r(66,89)$, and the stress axis genes $g r 1$ and $m r(90)$ in whole-body samples of fry from the two parental treatments (two to four replicates per brood) from two sampling time points (day 3 and 10). Hormones and receptors from the somatotropic axis are globally expressed in the body, especially in the bone and muscle tissues, and have multiple functions throughout the body (91). Genes related to the corticosteroid signaling including $g r 1$ and $m r$ are expressed in the brain and other tissues of the body, and whole-body samples have been previously used to characterize the stress axis of the earliest developmental stages of fish (92). We then compared expression levels between treatments. RNA extracted (Qiagen RNeasy Micro Kit) from whole-body samples of each fry was reverse transcribed using the HighCapacity RNA-to-cDNA Kit (Thermo Fisher Scientific) following manufacturer's instructions (SI Appendix). To assay expression of somatotropic and stress axis genes, available sequences from African cichlids were used to design primers for all but one candidate gene (SI Appendix, Materials and Methods and Table S2). Three reference genes, which were previously used in three fish species, were tested for normalization: $18 \mathrm{~s}$ (93) in $N$. pulcher (89), efa1 (94) in Oreochromis niloticus, and rpl13a in D. rerio (95). Among these genes, rp/13a was most stably expressed across experimental groups and therefore was used as reference gene in this study.

The reverse-transcribed complementary DNA (CDNA) was preamplified for the aforementioned amplicons of interest using the TaqMan PreAmp Master Mix (Applied Biosystems; Fluidigm protocol: PN100-5875C1). The samples were diluted fivefold (SI Appendix) and assayed for gene expression on the Flex Six IFC (Fluidigm) chip using the SsoFast EvaGreen Supermix with Low ROX (Bio-Rad) (Fluidigm protocol: PN100-7717B1). The chip was run on the BioMark system by Fluidigm that allows for great sensitivity and is well suited for quantification of genes from very small tissues. We quantified gene expression using the comparative $C_{\mathrm{T}}$ method [2- ${ }^{-\Delta \Delta C_{T}}$ (96)] and report fold change of the gene in question relative to $r p / 13 a$.

1. H. Schwabl, T. G. G. Groothuis, "Maternal effects on behavior" in Encyclopedia of Animal Behavior, M. D. Breed, J. Moore, Eds. (Elsevier, 2019), pp. 483-494.

2. T. Uller, Developmental plasticity and the evolution of parental effects. Trends Ecol. Evol. 23, 432-438 (2008).

3. T. A. Mousseau, C. W. Fox, The adaptive significance of maternal effects. Trends Ecol. Evol. 13, 403-407 (1998).

4. J. B. Wolf, E. D. Brodie III, J. M. Cheverud, A. J. Moore, M. J. Wade, Evolutionary consequences of indirect genetic effects. Trends Ecol. Evol. 13, 64-69 (1998).

5. T. G. Benton, S. J. Plaistow, A. P. Beckerman, C. T. Lapsley, S. Littlejohns, Changes in maternal investment in eggs can affect population dynamics. Proc. Biol. Sci. 272, 1351-1356 (2005).

6. S. Ruuskanen, B. Doligez, L. Gustafsson, T. Laaksonen, Long-term effects of yolk androgens on phenotype and parental feeding behavior in a wild passerine. Behav. Ecol. Sociobiol. 66, 1201-1211 (2012)

7. O. P. Love, P. O. Mcgowan, M. J. Sheriff, Maternal adversity and ecological stressors in natural populations: The role of stress axis programming in individuals, with implications for populations and communities. Funct. Ecol. 27, 81-92 (2013).
Statistical analysis. For statistical analyses we used R 2.14.1 (R Core Development Team, 2012) with the package Ime4 (97) and afex (98). Linear mixed models (LMMs) were run for models with a normally distributed error term. For all LMMs, Quantile-Quantile plots and residuals were visually examined to check normality assumptions. If necessary, dependent variables were transformed to meet the requirements of normality. All GLMMs (see $S I$ Appendix, Table S6 for details) were fitted with a log-link function (Poisson distribution). We tested for overdispersion in these models by comparing the residual deviance to the residual degrees of freedom; none of these models were overdispersed. For both LMMs and GLMMs, brood ID was included as a random factor, since multiple replicates from the same brood were analyzed. Significance testing was always based on deviance when removing respective terms from the model. The change in likelihood was compared to a $\chi^{2}$ distribution (likelihood ratio test). All models analyzed in this study are described in SI Appendix, Table S6 with their fixed and random factors, type of model, link functions, and any transformations, if applicable.

For experiment 1, LMMs were fitted to analyze treatment differences for egg weights and total amino acid content and concentration (with brood ID as a random factor), while for clutch size, a linear model (LM) was fitted.

For experiment 2, an LM was fitted to assess treatment effects on clutch size with treatment, maternal weight, and number of presentations and their interaction as fixed factors. LMMs were fitted to assess treatment differences for dry body weights at days 3 and 10 after adjusting for number of offspring present in the tanks ("density"). The LMM for response latencies was corrected for the angle of the focal relative to the dropping marble ( $S I A p$ pendix, Materials and Methods) and for focal length because the length influences burst swim speed (42). A GLMM was also fitted for feeding frequency after the marble drop, which included body condition of the focal fish (represented by Fulton's index [FI], a measure used frequently in fish; FI = body weight $[\mathrm{g}] /$ standard length $[\mathrm{cm}]^{3} \times 100$ ), because this parameter might influence hunger levels. Fold-change values of gene expression were analyzed by fitting LMMs using boxcox transformations (see SI Appendix, Table S6 for the transformation exponents). Additional models on lengths and weights at 3 mo, offspring survival at days 3 and 10, where survival was calculated as a percentage of the original clutch size, the probability to enter the shelter after the marble drop, and the frequency of shelter visits are described in SI Appendix, Table S6 and their results are given in SI Appendix, Table S4.

In these models, whenever multiple replicates from a brood were analyzed in models, brood ID was included as a random factor. All statistical results are presented with their estimates (Tables 1 and 2 and SI Appendix, Tables S4 and S5). All estimates presented in the tables are based on sum contrasts, where the intercept represents the overall mean of each factor and each estimate represents the difference between the intercept and factor level of interest. Main results of the experiments are presented in Tables 1 and 2 and additional results in SI Appendix, Tables S4 and S5 and Fig. S4.

Data Availability. Data have been deposited in Figshare, https://doi.org/10 6084/m9.figshare.15112773.v1 (99).

ACKNOWLEDGMENTS. We thank Urs Kämpfer for help with the amino acid quantification and Evi Zwygart and Markus Wymann for logistical support. Gene expression assays were possible with valuable input from Peter Nestorov, Aria Minder, and Silvia Kobel at the Genetic Diversity Centre ETH Zurich. We acknowledge constructive comments by the editor, two anonymous referees and by Michael Taborsky on an earlier draft of this manuscript. The study was funded by the Swiss National Science Foundation (Grant 31003A 156881 to B.T.). All procedures were conducted under the license 74/15 of the Veterinäramt Bern and adhered to the guidelines of the Association for the Study of Animal Behaviour.

8. M. J. Sheriff, O. P. Love, Determining the adaptive potential of maternal stress. Ecol. Lett. 16, 271-280 (2013).

9. D. J. Marshall, T. Uller, When is a maternal effect adaptive? Oikos 116, 1957-1963 (2007)

10. S. Via, Models of the evolution of phenotypic plasticity. Trends Ecol. Evol. 7, 63 (1992)

11. S. C. Stearns, Life history evolution: Successes, limitations, and prospects. Naturwissenschaften 87, 476-486 (2000)

12. M. R. Walsh, D. N. Reznick, Interactions between the direct and indirect effects of predators determine life history evolution in a killifish. Proc. Natl. Acad. Sci. U.S.A. 105, 594-599 (2008).

13. M. J. Sheriff, C. J. Krebs, R. Boonstra, The ghosts of predators past: Population cycles and the role of maternal programming under fluctuating predation risk. Ecology 91, 2983-2994 (2010).

14. F. Groenewoud et al., Predation risk drives social complexity in cooperative breeders. Proc. Natl. Acad. Sci. U.S.A. 113, 4104-4109 (2016)

15. D. Heg, Z. Bachar, L. Brouwer, M. Taborsky, Predation risk is an ecological constraint for helper dispersal in a cooperatively breeding cichlid. Proc. Biol. Sci. 271, 2367-2374 (2004). 
16. R. Tollrian, Predator-induced morphological defenses: Costs, life history shifts, and maternal effects in Daphnia pulex. Ecology 76, 1691-1705 (1995).

17. E. R. Giesing, C. D. Suski, R. E. Warner, A. M. Bell, Female sticklebacks transfer information via eggs: Effects of maternal experience with predators on offspring Proc. Biol. Sci. 278, 1753-1759 (2011).

18. A. Stratmann, B. Taborsky, Antipredator defences of young are independently determined by genetic inheritance, maternal effects and own early experience in mouthbrooding cichlids. Funct. Ecol. 28, 944-953 (2014).

19. K. E. McGhee, A. J. Barbosa, K. Bissell, N. A. Darby, S. Foshee, Maternal stress during pregnancy affects activity, exploration and potential dispersal of daughters in an invasive fish. Anim. Behav. 171, 41-50 (2021).

20. E. Bestion, A. Teyssier, F. Aubret, J. Clobert, J. Cote, Maternal exposure to predator scents: Offspring phenotypic adjustment and dispersal. Proc. Biol. Sci. 281, 20140701 (2014).

21. S. Cattelan et al., Maternal predation risk increases offspring's exploration but does not affect schooling behavior. Behav. Ecol. 31, 1207-1217 (2020).

22. K. E. McGhee, L. M. Pintor, E. L. Suhr, A. M. Bell, Maternal exposure to predation risk decreases offspring antipredator behaviour and survival in threespined stickleback. Funct. Ecol. 26, 932-940 (2012).

23. M. Seiter, P. Schausberger, Maternal intraguild predation risk affects offspring antipredator behavior and learning in mites. Sci. Rep. 5, 15046 (2015).

24. S. Feng, K. E. McGhee, A. M. Bell, Effect of maternal predator exposure on the ability of stickleback offspring to generalize a learned colour-reward association. Anim. Behav. 107, 61-69 (2015).

25. D. P. Roche, K. E. McGhee, A. M. Bell, Maternal predator-exposure has lifelong consequences for offspring learning in threespined sticklebacks. Biol. Lett. 8 932-935 (2012).

26. J. Freinschlag, P. Schausberger, Predation risk-mediated maternal effects in the twospotted spider mite, Tetranychus urticae. Exp. Appl. Acarol. 69, 35-47 (2016).

27. J. J. Storm, S. L. Lima, Mothers forewarn offspring about predators: A trans generational maternal effect on behavior. Am. Nat. 175, 382-390 (2010).

28. H. Neumeister, K. W. Whitaker, H. A. Hofmann, T. Preuss, Social and ecological regulation of a decision-making circuit. J. Neurophysiol. 104, 3180-3188 (2010).

29. R. C. Eaton, R. A. Bombardieri, D. L. Meyer, The Mauthner-initiated startle response in teleost fish. J. Exp. Biol. 66, 65-81 (1977).

30. A. Hecker, W. Schulze, J. Oster, D. O. Richter, S. Schuster, Removing a single neuron in a vertebrate brain forever abolishes an essential behavior. Proc. Natl. Acad. Sci. U.S.A. 117, 3254-3260 (2020)

31. M. I. McCormick, E. Fakan, B. J. M. Allan, Behavioural measures determine survivorship within the hierarchy of whole-organism phenotypic traits. Funct. Ecol. 32 958-969 (2018).

32. H. B. Nguyen, R. C. Bagot, J. Diorio, T. P. Wong, M. J. Meaney, Maternal care dif ferentially affects neuronal excitability and synaptic plasticity in the dorsal and ventral hippocampus. Neuropsychopharmacology 40, 1590-1599 (2015).

33. I. C. G. Weaver et al., Epigenetic programming by maternal behavior. Nat. Neurosci. 7, 847-854 (2004).

34. L. R. Stein, A. M. Bell, Paternal programming in sticklebacks. Anim. Behav. 95, 165-171 (2014).

35. A. M. Bell, K. E. McGhee, L. Stein, Effects of mothers' and fathers' experience with predation risk on the behavioral development of their offspring in threespined sticklebacks. Curr. Opin. Behav. Sci. 7, 28-32 (2016)

36. A. B. Rodgers, C. P. Morgan, N. A. Leu, T. L. Bale, Transgenerational epigenetic programming via sperm microRNA recapitulates effects of paternal stress. Proc. Nat/. Acad. Sci. U.S.A. 112, 13699-13704 (2015).

37. K. Gapp et al., Implication of sperm RNAs in transgenerational inheritance of the effects of early trauma in mice. Nat. Neurosci. 17, 667-669 (2014).

38. T. G. G. Groothuis, H. Schwabl, Hormone-mediated maternal effects in birds Mechanisms matter but what do we know of them? Philos. Trans. R. Soc. Lond. B Biol. Sci. 363, 1647-1661 (2008)

39. K. E. McGhee, R. T. Paitz, J. A. Baker, S. A. Foster, A. M. Bell, Effects of predation risk on egg steroid profiles across multiple populations of threespine stickleback. Sci Rep. 10, 5239 (2020).

40. L. A. Fuiman, A. F. Ojanguren, Fatty acid content of eggs determines antipredator performance of fish larvae. J. Exp. Mar. Biol. Ecol. 407, 155-165 (2011).

41. F. H. I. D. Segers, B. Taborsky, Egg size and food abundance interactively affect juvenile growth and behaviour. Funct. Ecol. 25, 166-176 (2011).

42. R. Schürch, B. Taborsky, The functional significance of buccal feeding in the mouthbrooding cichlid Tropheus moorii. Behaviour 142, 265-281 (2005).

43. J. A. Walker, C. K. Ghalambor, O. L. Griset, D. McKenney, D. N. Reznick, Do faster starts increase the probability of evading predators? Funct. Ecol. 19, 808-815 (2005)

44. A. M. Bennett, D. L. Murray, Maternal body condition influences magnitude of antipredator response in offspring. Proc. Biol. Sci. 281, 20141806 (2014).

45. S. Brooks, C. R. Tyler, J. P. Sumpter, Egg quality in fish: What makes a good egg? Rev. Fish Biol. Fish. 7, 387-416 (1997)

46. F. H. I. D. Segers, B. Taborsky, Juvenile exposure to predator cues induces a larger egg size in fish. Proc. Biol. Sci. 279, 1241-1248 (2012)

47. R. A. Ramasamy, B. J. M. Allan, M. I. McCormick, Plasticity of escape responses: Prior predator experience enhances escape performance in a coral reef fish. PLoS One 10 e0132790 (2015)

48. S. Fischer et al., Animated images as a tool to study visual communication: A case study in a cooperatively breeding cichlid. Behaviour 151, 1921-1942 (2014).

49. H. Neumeister et al., Socially induced plasticity in sensorimotor gating in the African cichlid fish Astatotilapia burtoni. Behav. Brain Res. 332, 32-39 (2017)

50. B. G. Dias, K. J. Ressler, Parental olfactory experience influences behavior and neural structure in subsequent generations. Nat. Neurosci. 17, 89-96 (2014).
51. M. Kavaliers, E. Choleris, Antipredator responses and defensive behavior: Ecological and ethological approaches for the neurosciences. Neurosci. Biobehav. Rev. 25, 577-586 (2001).

52. M. Milinski, R. Heller, Influence of a predator on the optimal foraging behaviour of sticklebacks (Gasterosteus aculeatus L.). Nature 275, 642-644 (1978).

53. B. Taborsky, E. Skubic, R. Bruintjes, Mothers adjust egg size to helper number in a cooperatively breeding cichlid. Behav. Ecol. 18, 652-657 (2007).

54. L. Brouwer, D. Heg, M. Taborsky, Experimental evidence for helper effects in a cooperatively breeding cichlid. Behav. Ecol. 16, 667-673 (2005)

55. M. G. Meekan, L. Fortier, Selection for fast growth during the larval life of Atlantic cod Gadus morhua on the Scotian shelf. Mar. Ecol. Prog. Ser. 137, 25-37 (1996).

56. M. A. J. Bergenius, M. G. Meekan, R. D. Robertson, M. I. McCormick, Larval growth predicts the recruitment success of a coral reef fish. Oecologia 131, 521-525 (2002).

57. S. Balshine et al., Correlates of group size in a cooperatively breeding cichlid fish (Neolamprologus pulcher). Behav. Ecol. Sociobiol. 50, 134-140 (2001).

58. S. Mukherjee, M. R. Heithaus, J. C. Trexler, J. Ray-Mukherjee, J. Vaudo, Perceived risk of predation affects reproductive life-history traits in Gambusia holbrooki, but not in Heterandria formosa. PLoS One 9, e88832 (2014)

59. K. Kasimatis, C. Riginos, A phylogenetic analysis of egg size, clutch size, spawning mode, adult body size, and latitude in reef fishes. Coral Reefs 35, 387-397 (2016).

60. G. Marteinsdottir, A. Steinarsson, Maternal influence on the size and viability of Iceland cod Gadus morhua eggs and larvae. J. Fish Biol. 52, 1241-1258 (1998).

61. A. Arukwe, A. Goksøyr, Eggshell and egg yolk proteins in fish: Hepatic proteins for the next generation: Oogenetic, population, and evolutionary implications of endocrine disruption. Comp. Hepatol. 2, 4 (2003)

62. M. C. Pannevis, D. F. Houlihan, The energetic cost of protein synthesis in isolated hepatocytes of rainbow trout (Oncorhynchus mykiss). J. Comp. Physiol. B 162, 393-400 (1992)

63. M. Reinecke et al., Growth hormone and insulin-like growth factors in fish: Where we are and where to go. Gen. Comp. Endocrinol. 142, 20-24 (2005).

64. C. A.-L. Leblanc, The Importance of Egg Size for the Diversity of Salmonids (Oregon State University, Corvallis, OR, 2011).

65. M. B. Eldridge, J. A. Whipple, M. J. Bowers, Bioenergetics and growth of striped bass, Morone saxatilis, embryos and larvae. Fish Bull. 80, 461-474 (1982).

66. F. H. I. D. Segers, G. Berishvili, B. Taborsky, Egg size-dependent expression of growth hormone receptor accompanies compensatory growth in fish. Proc. Biol. Sci. 279, 592-600 (2012)

67. K. Kotrschal, M. J. Van Staaden, R. Huber, Fish brains: Evolution and environmental relationships. Rev. Fish Biol. Fish. 8, 373-408 (1998).

68. M. V. Abrahams, A. Sutterlin, The foraging and antipredator behaviour of growthenhanced transgenic Atlantic salmon. Anim. Behav. 58, 933-942 (1999)

69. C. Håkonsrud Jensen et al., Hormonal adjustments to future expectations impact growth and survival in juvenile fish. Oikos 130, 41-51 (2021).

70. S. L. White, H. Volkoff, R. H. Devlin, Regulation of feeding behavior and food intake by appetite-regulating peptides in wild-type and growth hormone-transgenic coho salmon. Horm. Behav. 84, 18-28 (2016).

71. R. Bruintjes, M. Taborsky, Size-dependent task specialization in a cooperative cichlid in response to experimental variation of demand. Anim. Behav. 81, 387-394 (2011).

72. D. Heg, M. Taborsky, Helper response to experimentally manipulated predation risk in the cooperatively breeding cichlid Neolamprologus pulcher. PLoS One 5, e10784 (2010)

73. M. Taborsky, D. Limberger, Helpers in fish. Behav. Ecol. Sociobiol. 8, 143-145 (1981).

74. M. Taborsky, E. Hert, M. von Siemens, P. Stoerig, Social behaviour of Lamprologus species: Functions and mechanisms. Ann. K. Museum Midden-Afrika Zool. Wet. 251, 7-11 (1986).

75. B. C. Mommer, A. M. Bell, A test of maternal programming of offspring stress response to predation risk in threespine sticklebacks. Physiol. Behav. 122, 222-227 (2013).

76. H. Rauwerda et al., Mother-specific signature in the maternal transcriptome composition of mature, unfertilized zebrafish eggs. PLoS One 11, e0147151 (2016).

77. A. Konings, Tanganyika Cichlids in Their Natural Habitat (Cichlid Press, 1998).

78. M. Taborsky, "Cichlid fishes: A model for the integrative study of social behavior" in Cooperative Breeding in Vertebrates: Studies of Ecology, Evolution, and Behavior, W. D. Koenig, J. L. Dickinson, Eds. (Cambridge University Press, 2016), pp. 272-293.

79. M. Watve, S. Prati, B. Taborsky, Simulating more realistic predation threat using attack playbacks. PeerJ 7, e8149 (2019).

80. S. Fischer, E. Oberhummer, F. Cunha-Saraiva, N. Gerber, B. Taborsky, Smell or vision? The use of different sensory modalities in predator discrimination. Behav. Ecol. Sociobiol. 71, 143 (2017).

81. T. Baumann et al., Ctenidins: Antimicrobial glycine-rich peptides from the hemocytes of the spider Cupiennius salei. Cell. Mol. Life Sci. 67, 2787-2798 (2010).

82. A. R. Reddon, D. Balk, S. Balshine, Probing aggressive motivation during territorial contests in a group-living cichlid fish. Behav. Processes 92, 47-51 (2013)

83. O. Friard, M. Gamba, BORIS: A free, versatile open-source event-logging software for video/audio coding and live observations. Methods Ecol. Evol. 7, 1325-1330 (2016).

84. J. I. Johnsson, B. T. Björnsson, Growth hormone increases growth rate, appetite and dominance in juvenile rainbow trout, Oncorhynchus mykiss. Anim. Behav. 48, 177-186 (1994).

85. M. Codina et al., Metabolic and mitogenic effects of IGF-II in rainbow trout (Oncorhynchus mykiss) myocytes in culture and the role of IGF-II in the PI3K/Akt and MAPK signalling pathways. Gen. Comp. Endocrinol. 157, 116-124 (2008).

86. Q. P. Vong, K. M. Chan, C. H. K. Cheng, Quantification of common carp (Cyprinus carpio) IGF-I and IGF-II mRNA by real-time PCR: Differential regulation of expression by GH. J. Endocrinol. 178, 513-521 (2003)

87. E. R. De Kloet, E. Vreugdenhil, M. S. Oitzl, M. Joëls, Brain corticosteroid receptor balance in health and disease. Endocr. Rev. 19, 269-301 (1998). 
88. L. Jacobson, R. Sapolsky, The role of the hippocampus in feedback regulation of the hypothalamic-pituitary-adrenocortical axis. Endocr. Rev. 12, 118-134 (1991).

89. C. Nyman, S. Fischer, N. Aubin-Horth, B. Taborsky, Effect of the early social environment on behavioural and genomic responses to a social challenge in a cooperatively breeding vertebrate. Mol. Ecol. 26, 3186-3203 (2017).

90. B. Taborsky, L. Tschirren, C. Meunier, N. Aubin-Horth, Stable reprogramming of brain transcription profiles by the early social environment in a cooperatively breeding fish. Proc. Biol. Sci. 280, 20122605 (2012).

91. E. Jönsson, B. T. Björnsson, Physiological functions of growth hormone in fish with special reference to its influence on behaviour. Fish. Sci. 68, 742-748 (2002).

92. A. Tsalafouta et al., Ontogenesis of the HPI axis and molecular regulation of the cortisol stress response during early development in Dicentrarchus labrax. Sci. Rep. 4, 5525 (2014).

93. C. M. O'Connor, S. E. Marsh-Rollo, S. C. Ghio, S. Balshine, N. Aubin-Horth, Is there convergence in the molecular pathways underlying the repeated evolution of sociality in African cichlids? Horm. Behav. 75, 160-168 (2015).

94. E. Wang et al., Evaluation and selection of appropriate reference genes for real-time quantitative PCR analysis of gene expression in Nile tilapia (Oreochromis niloticus) during vaccination and infection. Int. J. Mol. Sci. 16, 9998-10015 (2015).
95. R. Tang, A. Dodd, D. Lai, W. C. McNabb, D. R. Love, Validation of zebrafish (Danio rerio) reference genes for quantitative real-time RT-PCR normalization. Acta Biochim. Biophys. Sin. (Shanghai) 39, 384-390 (2007).

96. K. J. Livak, T. D. Schmittgen, Analysis of relative gene expression data using real-time quantitative PCR and the 2(- $\Delta \Delta C(T))$ method. Methods 25, 402-408 (2001).

97. D. Bates, M. Mächler, B. Bolker, Ime4: Linear Mixed-Effects Models Using S4 Classes (R Package version 0.999375-42, 2011). https://cran.r-project.org/web/packages/lme4/ index.html. Accessed 31 August 2021.

98. H. Singmann, B. Bolker, J. Westfall, afex: Analysis of Factorial Experiments (R package version 0.15-2, 2015). http://CRAN.R-project.org/package=afex. Accessed 31 August 2021.

99. S. Sharda, T. Zuest, M. Erb, B. Taborsky, Data for experiments used in the paper titled "Predator-induced maternal effects determine adaptive antipredator behaviors via egg composition." Figshare. https://doi.org/10.6084/m9.figshare.15112773.v1. Deposited 8 May 2021.

100. B. C. Mommer, A. M. Bell, Maternal experience with predation risk influences genome-wide embryonic gene expression in threespined sticklebacks (Gasterosteus aculeatus). PLoS One 9, e98564 (2014).

101. M. Reyes-Contreras, G. Glauser, D. J. Rennison, B. Taborsky, Early-life manipulation of cortisol and its receptor alters stress axis programming and social competence. Philos. Trans. R. Soc. Lond. B Biol. Sci. 374, 20180119 (2019). 